\title{
Effects of Low-Polarizability Perturbers on the Cadmium Intercombination Line
}

\author{
A. Bielski, D. Lisak, R.S. Trawiński and J. Szudy \\ Institute of Physics, Nicholas Copernicus University \\ Grudziądzka 5/7, 87-100 Toruń, Poland
}

(Received October 14, 2002)

\begin{abstract}
Using a laser-induced fluorescence method a detailed analysis of profiles of the ${ }^{114} \mathrm{Cd} 326.1 \mathrm{~nm}$ line perturbed by neon and helium was performed which revealed deviations from the ordinary Voigt profile. These deviations are shown to be consistent with fits of experimental profiles to an asymmetric Voigt profile. Coefficients of the pressure broadening, shift and collision-time asymmetry are determined and compared with those calculated in the adiabatic approximation for different interaction potentials.
\end{abstract}

PACS numbers: $32.70 .-\mathrm{n}, 33.70 .-\mathrm{w}, 34.20 .-\mathrm{b}$

\section{Introduction}

Extensive studies on noble-gas-induced broadening and shift of atomic spectral lines have revealed different behaviour of heavy, i.e. high-polarizability noble gases such as $\mathrm{Ar}, \mathrm{Kr}$ and $\mathrm{Xe}$ and the light gases such as $\mathrm{He}$ and $\mathrm{Ne}$ characterized by small value of polarizability $\xi[1,2]$. For high-polarizability noble gases the main features of the broadening and shift phenomena can be, in principle, explained in terms of a van der Waals potential. These gases always produce a red shift of the line and the asymmetry of the line shape - if it appears - is such that the intensity in the long-wavelength side of the line is higher than that in the short-wavelength one (red asymmetry). Contrary to that the effects produced on spectral lines by low-polarizability perturbing gases are not so clearly defined. Helium, for instance, was considered for many years as a gas producing a blue shift and symmetric profiles of spectral lines [1,2]. Nevertheless, Lewis and his co-workers have found that in the case of the calcium $422.7 \mathrm{~nm}$ line helium produces a red shift and a blue asymmetry of the profile [3-5]. Recently, however, 
Romalis et al. [6] have observed both the blue asymmetry and the blue shift of the resonance doublet of rubidium perturbed by the ${ }^{4} \mathrm{He}$ and ${ }^{3} \mathrm{He}$ isotopes. Much more obscure behaviour was observed by many researchers for neon used in the role of perturbing gas. Chen and Takeo long time ago [1] have emphasized that the shift produced by neon is usually very small and may be directed either towards blue or towards red. A puzzling behaviour of neon was also observed for Rydberg spectral lines associated with two-photon transitions in sodium [7]. It is generally believed that the case of broadening and shift induced by Ne-atoms must be treated with extreme caution. This is due to the fact that both theoretical and experimental values of the electron-scattering length $L$ for neon reported in various papers differ markedly from each other. It is important to note that all existing in literature values of $L$ (both calculated theoretically and determined experimentally) for the Ne-atom are positive $(L>0)$ although rather small. The consequence of this is that according to the Fermi-Omont theory [8,9] for perturbers with $L>0$ optical lines involving Rydberg or quasi-Rydberg states should be shifted towards blue. Contrary to that, in some cases, e.g. for two quasi-Rydberg spectral lines of argon involving $4 d$-states perturbed by neon, a red shift was observed [10]. On the other hand, for non-Rydberg lines the neon-induced shift can be either toward blue as for instance in the case of $8{ }^{2} P_{3 / 2}-7{ }^{2} S_{1 / 2}(655 \mathrm{~nm})$ and $8^{2} P_{1 / 2}-7{ }^{2} S_{1 / 2}$ $(671.4 \mathrm{~nm})$ spectral lines of thallium [11] or towards red as in the case of the $7 S_{1 / 2}-6 P_{3 / 2}(535.0 \mathrm{~nm})$ green line $[12,13]$ and the $7 S_{1 / 2}-6 P_{1 / 2}(377.68 \mathrm{~nm})$ resonance line $[13,14]$ of thallium.

In earlier work in this laboratory [15-17] high-resolution studies of pressure broadening, shift and asymmetry of the cadmium intercombination line $326.1 \mathrm{~nm}$ $\left(5{ }^{1} S_{0}-5{ }^{3} P_{1}\right)$ perturbed by $\mathrm{Xe}[15], \mathrm{Kr}[16]$ and $\mathrm{Ar}$ [17] were reported. They were performed using a laser-induced fluorescence (LIF) method. A good signal-to-noise ratio and negligible instrumental profile enabled us to fit the experimental line shapes to theoretical ones in considerable detail and determine in very good accuracy the values of pressure broadening, shift and asymmetry parameters. For all these three high-polarizability noble gases ( $\mathrm{Ar}, \mathrm{Kr}, \mathrm{Xe}$ ) and also for high polarizability molecular gases such as $\mathrm{N}_{2}$ and $\mathrm{CH}_{4}$ we have found a red shift and red asymmetry of the $326.1 \mathrm{~nm}$ Cd line confirming thus a general feature of these perturbers already observed in earlier experiments on radiating atoms other than cadmium (cf. $[1,2,18]$ ).

In this paper we report results of precise measurements of the profiles of the $326.1 \mathrm{~nm} \mathrm{Cd}$ line perturbed by low-polarizability noble gases (He and $\mathrm{Ne}$ ). Using the LIF method we performed very careful analysis of the influence of He and $\mathrm{Ne}$ on the broadening and shift of this line. Since the neon-induced shift was found to be very small we focused particular attraction on the accuracy of the shift measurements. In the case of perturbation by helium we encountered severe difficulties caused by the helium permeation through the walls of quartz fluorescence cells. It is one of the purposes of this paper to present details of 
our experimental procedure which enabled us to carry out measurements on the $\mathrm{Cd}-\mathrm{He}$ system in such a way that helium diffusion through quartz does not lead to noticeable errors.

The present paper is aimed toward the completion of investigations of noble-gas-induced broadening and shift effects on the $\mathrm{Cd}$ intercombination line. Together with the promising progress in the theoretical " $a b$ initio" calculations of the Cd-noble-gas-atom interaction potentials [19] and recent experiments on Cd-noble-gas excimers created in supersonic expansion [20] this should lead to a better understanding of these effects.

\section{Experiment}

The experimental apparatus and methods of analysis were similar to those described in previous papers from this laboratory [15-17, 21]. The measurements of the shape of the $\mathrm{Cd} 326.1 \mathrm{~nm}$ line perturbed either by neon or by helium were performed by means of the laser-induced fluorescence (LIF) technique using the ${ }^{114} \mathrm{Cd}$ isotope which enabled us to avoid the hyperfine and isotopic effects as well as to neglect the role of line-mixing asymmetry. The cadmium fluorescence was registered with a digital laser spectrometer [21] which consisted of an actively stabilized single-frequency Coherent CR 899-21 ring dye laser equipped with intracavity frequency doubler operating on DCM dye pumped by Coherent Innova 400-15 argon-ion laser. To obtain the profile of the pressure broadened line, the doubled frequency of the ring laser was scanned up to $60 \mathrm{GHz}$ while the fluorescence was monitored at a fixed wavelength $326.1 \mathrm{~nm}$. The intensity of the fluorescence signal was measured at a right angle to the laser beam with an EMI $6256 \mathrm{~A}$ photomultiplier working in the photon counting mode and thermoelectrically cooled to minimize the dark current. Photon counting was performed by an electronic system built in the CAMAC Standard [22]. The frequency-doubled ring laser beam incident on the fluorescence cell was linearly polarized in the vertical direction and the collection optics arm, perpendicular to the ring laser beam direction contained a linear polarizer set at the "magic angle" (rotated $54.7^{\circ}$ from the vertical), so that the collection optics system was insensitive to effects due to anisotropy of fluorescence (see e.g. [3, 23]). All the data, fluorescence signal, frequency-doubled ring laser output power, and the data needed for frequency calibration such as the transition peaks of a confocal Fabry-Perot interferometer (FPI) with a free-spectral range of $1.5 \mathrm{GHz}$ as well as the absorption spectrum of $\mathrm{I}_{2}$-molecules in the $100 \mathrm{~cm}$ long iodine absorption cell operated at the temperature $35^{\circ} \mathrm{C}$ were acquired with a $\mathrm{PC}$ computer for further evaluation. Frequency calibration of the ring laser was performed using its fundamental (not doubled) line which was directed to an FPI and $\mathrm{I}_{2}$-cell. 


\section{Line-shape analysis}

It has been well established both theoretically [24-26] and experimentally $[3-5]$ that for systems with small values $\alpha$ of the ratio of perturber and emitter masses $\alpha=m_{\mathrm{p}} / m_{\mathrm{e}}$ the correlation between the Doppler and collisional broadening can be neglected. In the case of $\mathrm{Cd}-\mathrm{He}$ and $\mathrm{Cd}-\mathrm{Ne}$ systems these ratios are equal to 0.035 and 0.18 , respectively. This means that both for $\mathrm{Cd}-\mathrm{He}$ and $\mathrm{Cd}-\mathrm{Ne}$ the Doppler broadening can be treated as a phenomenon statistically independent of collisional effects. The shape $I_{\mathrm{D}}(\widetilde{\nu})$ of the Doppler broadened line can then be described by a Gaussian distribution

$$
I_{\mathrm{D}}(\widetilde{\nu})=\frac{2 \sqrt{\ln 2}}{\gamma_{\mathrm{D}} \sqrt{\pi}} \exp \left[-\frac{4 \ln 2}{\gamma_{\mathrm{D}}^{2}}\left(\widetilde{\nu}-\widetilde{\nu}_{0}\right)^{2}\right]
$$

where $\widetilde{\nu}_{0}$ is the unperturbed wave number, $\gamma_{\mathrm{D}}$ is the Doppler (FWHM) width given by

$$
\gamma_{\mathrm{D}}=\frac{\widetilde{\nu}_{0}}{\pi} \sqrt{\frac{2 k_{\mathrm{B}} \ln 2}{m_{\mathrm{e}}} T},
$$

with $k_{\mathrm{B}}$ being Boltzmann's constant, and $T$ - the temperature.

The line shape caused by collisions of the emitting atoms with perturbers depends on whether the radiation emitted during a collision time is taken into account or not. In the so-called impact limit when this radiation is neglected which is equivalent to the assumption that the duration of each collision is negligibly short compared with the time between collisions, the collisional line shape can be described by a Lorentzian profile. The resulting line shape usually called the Voigt profile (VP) caused by the Doppler and collision effect treated as two statistically independent phenomena can then be presented in the form of a convolution of the Lorentzian and Gaussian distributions. If, however, the finite duration of collisions is taken into account, then - in the first approximation - the collisional line shape $I_{C}(\widetilde{\nu})$ can be presented as a sum of the Lorentzian and dispersion profiles $[27-30]$ :

$$
I_{\mathrm{C}}(\widetilde{\nu})=\frac{\left(\gamma_{\mathrm{L}} / 2\right)+\chi\left(\widetilde{\nu}-\widetilde{\nu}_{0}-\Delta\right)}{\left(\widetilde{\nu}-\widetilde{\nu}_{0}-\Delta\right)^{2}+\left(\gamma_{\mathrm{L}} / 2\right)^{2}}
$$

where $\gamma_{\mathrm{L}}, \Delta$, and $\chi$ denote the Lorentzian (FWHM) width, pressure shift and collision-time asymmetry parameter, respectively. The resulting line shape $I_{\mathrm{AVP}}(\widetilde{\nu})$ can then be written as a convolution

$$
I_{\mathrm{AVP}}(\widetilde{\nu})=I_{\mathrm{C}}(\widetilde{\nu}) \otimes I_{\mathrm{D}}(\widetilde{\nu})
$$

Following Lewis and his co-workers [3-5] such a convolution is referred to as the "asymmetric Voigt profile" (AVP). In the impact limit i.e. when the duration of collisions is assumed to be negligibly small, the asymmetry factor vanishes $(\chi=0)$ and the AVP becomes identical to the VP. In the present work we have fitted both the AVP and VP profiles to our experimental shapes of the $326.1 \mathrm{~nm}$ line perturbed 
by He and Ne. The best fit procedure was performed using a least-squares algorithm for nonlinear parameters given by Marquardt [31]. Our numerical fit to the VP profile allowed three parameters to vary: the Doppler width $\gamma_{\mathrm{D}}$, the Lorentzian width $\gamma_{\mathrm{L}}$ and the line shift $\Delta$. For the AVP profile we also fitted the asymmetry parameter $\chi$. The best fit procedure performed for various pressures of the perturbing gas enabled us to determine dependences of the line shape parameters on the density number $N$ of perturbers.

\section{Measurements involving neon}

The measurements of the shape of the $326.1 \mathrm{~nm}$ Cd line perturbed by neon were carried out using the ${ }^{114} \mathrm{Cd}$ isotope which was placed in a fluorescence cell made of quartz. The cell which was cylindrical in shape, $11.0 \mathrm{~cm}$ long and $3.0 \mathrm{~cm}$ in diameter has a sidearm $16.0 \mathrm{~cm}$ long and $1.0 \mathrm{~cm}$ in diameter. The ${ }^{114} \mathrm{Cd}$ isotope was redistilled under vacuum and distilled into the sidearm of the cell which had previously been baked many times in a flame; then at a room temperature the cell was filled with neon whose pressure could vary between 5 and 442 Torr. The sidearm was situated in a special multisection oven enabling the independent temperature stabilization of the cell and its sidearm up to $1 \mathrm{~K}$. During the measurements the sidearm temperature which determined the cadmium vapour pressure in the cell was maintained constant at $440 \mathrm{~K}$, while the cell temperature was kept at $724 \mathrm{~K}$.

Figure 1a shows an example of the shape of the $326.1 \mathrm{~nm}{ }^{114} \mathrm{Cd}$ line perturbed by $\mathrm{Ne}$ at pressure of 292 Torr (at room temperature). The circles are experimental points, while the solid line represents the best fit to the asymmetric Voigt profile (AVP). We have also attempted to fit our data to the ordinary Voigt profile (VP). In order to examine the quality of the fits we used the so-called weighted residuals of the intensities defined by

$$
D_{\sigma}(\widetilde{\nu})=\frac{I_{\exp }(\widetilde{\nu})-I_{\text {theor }}(\widetilde{\nu})}{u(\widetilde{\nu})},
$$

where $I_{\text {exp }}(\widetilde{\nu})$ denotes measured intensity at wave number $\widetilde{\nu}, I_{\text {theor }}(\widetilde{\nu})$ is the theoretical (fitted) intensity assumed to be given either in the form of the AVP or VP profiles, and $u(\widetilde{\nu})$ is the uncertainty of the measured signal. In Fig. 1b we plotted these residuals $\left(D_{\sigma}^{\mathrm{VP}}(\widetilde{\nu})\right)$ for the case when $I_{\text {theor }}(\widetilde{\nu})$ was given by the VP profile, i.e. when the influence of the finite duration of collisions is neglected and no asymmetry of the profile is expected. We can see systematic departures of $D_{\sigma}(\widetilde{\nu})$ from zero in the line core $\left(\widetilde{\nu} \approx \widetilde{\nu}_{0}\right)$ as well as on line wings which can be regarded as a manifestation of the line asymmetry.

Figure 1c shows the residuals $\left(D_{\sigma}^{\operatorname{AVP}}(\widetilde{\nu})\right)$ for the case when $I_{\text {theor }}(\widetilde{\nu})$ is given by the AVP. In contrast to Fig. 1b, in this case the values of the residuals are spread uniformly about zero which confirms the good quality of the fit. We can thus conclude that in the case of $\mathrm{Cd}-\mathrm{Ne}$ the asymmetry caused by the finite collision 


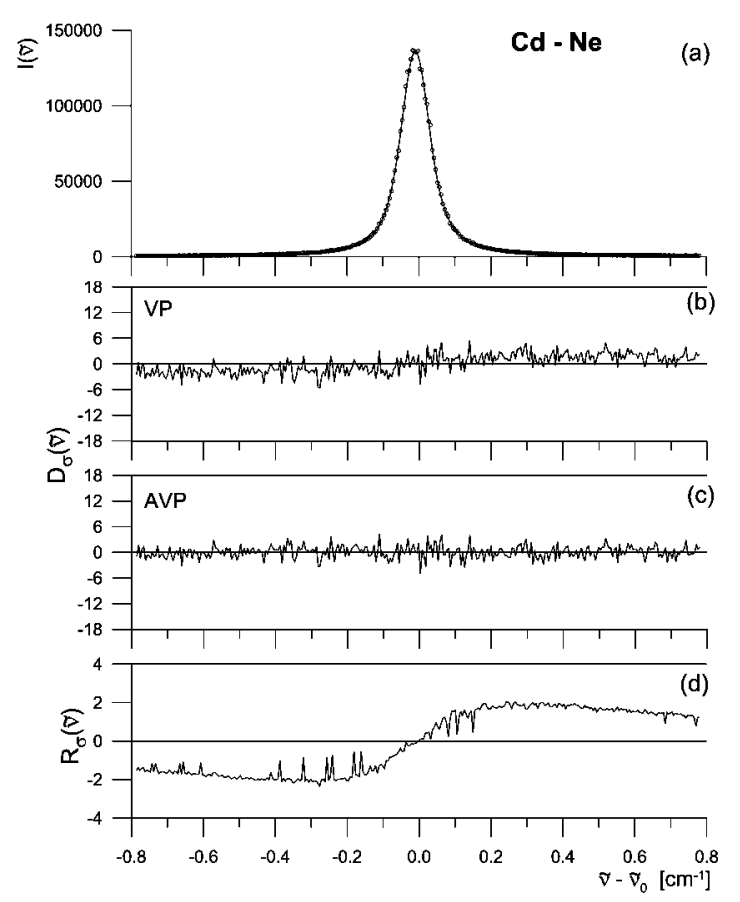

Fig. 1. The shape of the Cd $326.1 \mathrm{~nm}$ line perturbed by neon at pressure 292 Torr: (a) experimental points together with the best-fit AVP (full curve), (b) and (c) weighted residuals $D_{\sigma}(\widetilde{\nu})$ between experimental and fitted VP and AVP profiles, respectively, (d) differences $R(\widetilde{\nu})$.

time has a noticeable influence on the shape of the $326.1 \mathrm{~nm}$ line. This conclusion is particularly well illustrated in Fig. 1d, where the differences

$$
R_{\sigma}(\widetilde{\nu})=D_{\sigma}^{\mathrm{AVP}}(\widetilde{\nu})-D_{\sigma}^{\mathrm{VP}}(\widetilde{\nu})
$$

of the residuals shown in Fig. $1 \mathrm{~b}$ and $\mathrm{c}$ are plotted as a function of $\widetilde{\nu}-\widetilde{\nu}_{0}$. As it is seen, $R_{\sigma}(\widetilde{\nu})$ function has a typical dispersion form corresponding to the dispersion correction to the Lorentzian profile in accordance with Eq. (3) and with theoretical expectations [2-5, 27-29].

Figure 2 shows the values of the Doppler width $\gamma_{\mathrm{D}}$ of the $326.1 \mathrm{~nm} \mathrm{Cd}$ line plotted as a function of the neon pressure. The full circles are the experimental points of $\gamma_{\mathrm{D}}$ determined from the best fit of experimental profiles to the AVP profile. As can be seen, there is practically no dependence of the Gaussian width on the neon pressure. The average Gaussian width is found to be $\overline{\gamma_{\mathrm{D}}}=55(2) \times 10^{-3} \mathrm{~cm}^{-1}$. This means that $\gamma_{\mathrm{D}}$ can be really identical with the Doppler width, whose theoretical value (Eq. (2) for the cell temperature $724 \mathrm{~K}$ is equal to $55.4 \times 10^{-3} \mathrm{~cm}^{-1}$. According to Berman [32] and Ward et al. [24] the lack of the dependence of the experimentally determined values of $\gamma_{\mathrm{D}}$ from the AVP (or VP) fits on the perturb- 


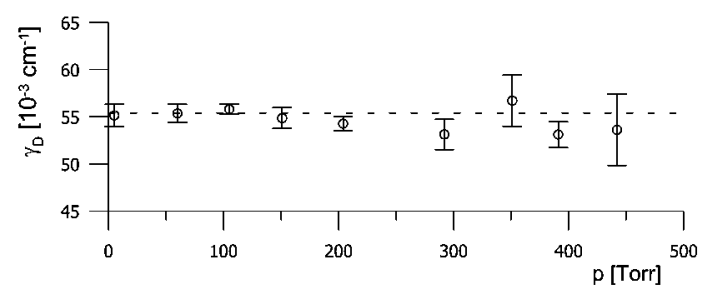

Fig. 2. Plot of the Doppler width $\gamma_{\mathrm{D}}$ of the $326.1 \mathrm{~nm}$ Cd line perturbed by neon against the pressure of $\mathrm{Ne}$. The widths $\gamma_{\mathrm{D}}$ are determined from the best fit of the AVP to the experimental data. Dashed line - Doppler width calculated from the cell temperature $(724 \mathrm{~K})$. Error bars indicate the value of the standard uncertainty.

ing gas pressure can be treated as an evidence that the correlation between the Doppler and collisional broadening does not play any role.

Figure 3 shows the plots of the Lorentzian width $\gamma_{\mathrm{L}}$ and shift $\Delta$ determined from the best fit of $I_{\mathrm{AVP}}(\widetilde{\nu})$ to our experimental profiles against the density number $N$ of neon. As it is seen the shift is toward the red (minus sign) and both $\gamma_{\mathrm{L}}$ and $\Delta$ are linearly dependent on the density. From the slopes of these linear dependencies the pressure broadening $\beta=\gamma_{\mathrm{L}} / N$ and shift $\delta=\Delta / N$ coefficients were determined and listed in Table I.

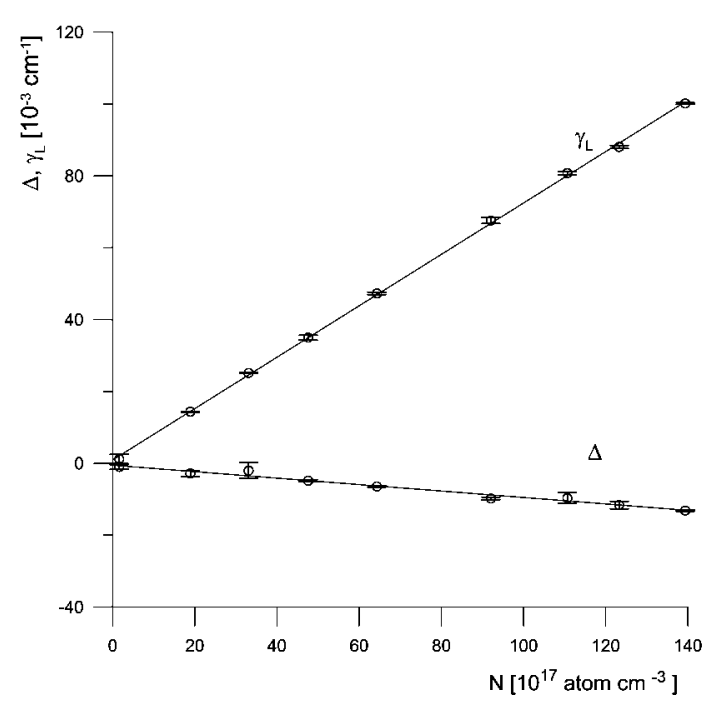

Fig. 3. Plot of the Lorentzian width $\gamma_{\mathrm{L}}$ and shift $\Delta$ of the $326.1 \mathrm{~nm} \mathrm{Cd}$ line determined from the best fit of the AVP to the experimental data against the neon density $N$. Error bars indicate the value of the standard uncertainty. 
TABLE I

Comparison of experimental values of the $\beta, \delta$ (in units $10^{-20} \mathrm{~cm}^{-1} /$ at. $\mathrm{cm}^{-3}$ ) and $\kappa$ (in units $10^{-21} /$ at. $\mathrm{cm}^{-3}$ ) coefficients for $\mathrm{Cd}-\mathrm{Ne}$ system with those calculated for different interatomic potentials. For experimental data the values of standard uncertainty are given.

\begin{tabular}{l|c|c|c}
\hline \hline Experimental values & $\beta_{\text {exp }}$ & $\delta_{\text {exp }}$ & $\kappa_{\exp }$ \\
\hline This work & $0.715(4)$ & $-0.090(5)$ & $0.17(7)$ \\
Bielski et al. [22] & $0.70(2)$ & $-0.13(2)$ & - \\
\hline Calculated values & $\beta_{\text {calc }}$ & $\delta_{\text {calc }}$ & $\kappa_{\text {calc }}$ \\
\hline Czuchaj-Stoll [19] & 0.750 & -0.142 & -0.025 \\
Morse & 0.734 & -0.063 & 0.06 \\
Lennard-Jones (SC) & 0.783 & 0.048 & 0.07 \\
van der Waals (H-F) & 0.828 & -0.301 & -0.59 \\
van der Waals (CA) & 0.644 & -0.234 & -0.20
\end{tabular}

Figure 4 shows the plot of the asymmetry parameter $\chi$ determined from the best fit of $I_{\mathrm{AVP}}(\widetilde{\nu})$ to our experimental profiles, against the density number $N$. As it is seen, the asymmetry parameter is linearly dependent on the density. From the slope of the best fit straight line the asymmetry coefficient $\kappa=\chi / N$ was determined (Table I).

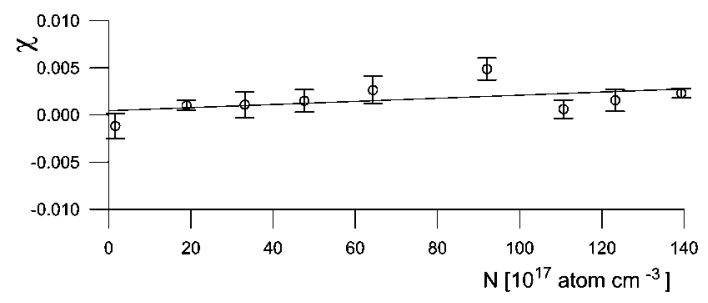

Fig. 4. Plot of the asymmetry parameter $\chi$ determined from the best fit of AVP to the experimental data against the neon density $N$. Error bars indicate the value of the standard uncertainty.

It should be emphasized that while the neon-induced shift of the $326.1 \mathrm{~nm}$ $\mathrm{Cd}$ line is towards red $(\Delta<0)$, the collision-time asymmetry coefficients for $\mathrm{Cd}-\mathrm{Ne}$ are positive $(\chi>0)$. This means that - as is clearly seen in Fig. 1c - the blue wing of this line has a higher intensity than the red one ("blue asymmetry"). Such a behaviour is in contrast to high-polarizability noble gases ( $\mathrm{Ar}, \mathrm{Kr}, \mathrm{Xe}$ ) which produce the red shift and the red asymmetry $(\chi<0)$ of the $\mathrm{Cd} 326.1 \mathrm{~nm}$ line [15-18].

The experimental values of pressure broadening $\beta$, shift $\delta$ and asymmetry $\kappa$ coefficients determined in the course of the present work are listed in Table I. In a 
previous work [22] the $\beta$ and $\delta$ coefficients for the $326.1 \mathrm{~nm}{ }^{114} \mathrm{Cd}$ line perturbed by Ne were determined using a classical spectroscopy technique, i.e. by means of a pressure scanned Fabry-Perot interferometer for the cell temperature $T=468 \mathrm{~K}$. These values are listed in Table I where they are marked "Bielski et al. [22]".

As can be seen from Table I, there is a good agreement between the values of $\beta$ and $\delta$ coefficients determined from different experiments performed for different temperatures. Our two independent experiments show that for the $326.1 \mathrm{~nm}$ $\mathrm{Cd}$ line perturbed by Ne the line shape parameters are independent of the temperature. This result is in agreement with our LIF [15-17] and interferometric [33-35] measurements and those performed by Dietz et al. [36], showing that for $\mathrm{Cd}-\mathrm{Xe}, \mathrm{Cd}-\mathrm{Kr}$ and $\mathrm{Cd}-\mathrm{Ar}$ systems no temperature dependencies of the line shape parameters were found.

\section{Measurements involving helium}

\subsection{Helium permeation through quartz}

In a set of preliminary LIF measurements of the shape of the $326.1 \mathrm{~nm} \mathrm{Cd}$ line perturbed by helium, we observed a specific effect which did not occur in the case of perturbation by neon and remaining noble gases [15-17]. The essence of this effect is illustrated in Fig. 5, which shows the values of the Lorentzian width $\gamma_{\mathrm{L}}$ of the helium-perturbed $326.1 \mathrm{~nm}$ Cd line determined from the fits to AVP profile of the experimental data obtained for the same fluorescence cell but at different time. All the data shown in Fig. 5 were obtained for the cell with the initial pressure of helium equal to 405 Torr. This is the pressure measured at room temperature at which the cell was filled with helium. Then the cell was situated in the oven and heated until the cell temperature was stabilized. After the cell temperature was stabilized within $\pm 1 \mathrm{~K}$ at $724 \mathrm{~K}$ we began the first measurement of the profile which lasted about 110 minutes. In Fig. 5 the numbers on the abscissa represent the time which passed since the beginning of the first measurement in minutes. As it is seen from Fig. 5, the Lorentzian widths $\gamma_{\mathrm{L}}$ determined in three subsequent measurements done at the same fluorescence cell decrease with the time passed since the first measurement. The value of $\gamma_{\mathrm{L}}$ determined at the second measurement which began immediately after the first one was completed was found to be $2.5 \%$ smaller than that in the first experiment. The same $2.5 \%$ decrease in $\gamma_{\mathrm{L}}$ was found in the third measurement which was completed about 330 minutes after the first one began.

Figure 6 shows the long-term changes of $\gamma_{\mathrm{L}}$ for the fluorescence cell filled at room temperature with 350 Torr of helium and then situated in the oven and heated to temperature $724 \mathrm{~K}$. Here the numbers on the abscissa (in hours) represent the time which passed since the beginning of heating; the heating time was equal to $12 \mathrm{~h}$. The points shown in Fig. 6 represent the experimental values of $\gamma_{\mathrm{L}}$ determined in five measurements performed after 12, 14, 30, 50, and $360 \mathrm{~h}$. The 


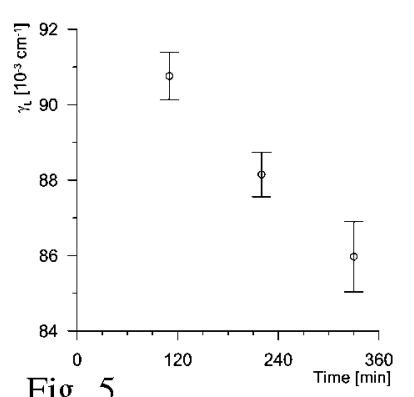

Fig. 5

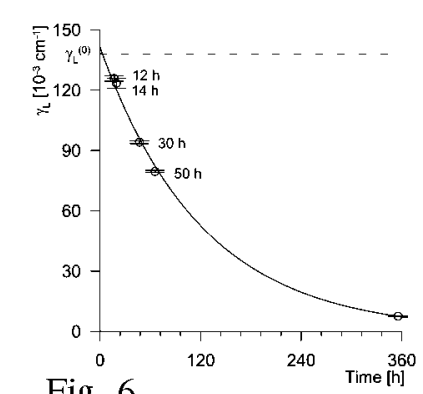

Fig. 6

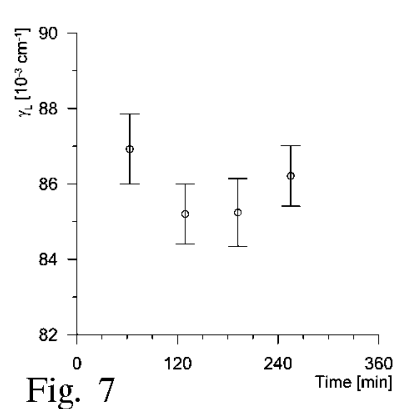

Fig. 7

Fig. 5. Plot of the Lorentzian width $\gamma_{\mathrm{L}}$ of the helium-perturbed $326.1 \mathrm{~nm} \mathrm{Cd}$ line determined from the best fits to AVP profile of the experimental data obtained for the same fluorescence cell but at different time. The numbers on the abscissa represent the time which passed since the beginning of the first measurement.

Fig. 6. The long-term changes of $\gamma_{\mathrm{L}}$ for the fluorescence cell filled at room temperature with 350 Torr of helium and then heated to temperature $724 \mathrm{~K}$. The numbers on the abscissa represent the time which passed since the beginning of heating.

Fig. 7. The values of the Lorentzian width $\gamma_{\mathrm{L}}$ determined for temperature $439 \mathrm{~K}$ from subsequent measurements of the shape of the $326.1 \mathrm{~nm} \mathrm{Cd}$ line perturbed by 230 Torr of helium. The numbers on the abscissa represent the time which passed since the beginning of the first measurement.

solid line shown in Fig. 6 is the best fit of these five experimental points to an experimentally decreasing function. The extrapolated value $\gamma_{\mathrm{L}}^{(0)}=0.14 \mathrm{~cm}^{-1} \mathrm{cor}-$ responding to the beginning of cell heating agrees with the value $\gamma_{\mathrm{L}}=0.137 \mathrm{~cm}^{-1}$ recalculated for the helium pressure 350 Torr using the value of the pressure broadening coefficient $\beta=1.22 \times 10^{-20} \mathrm{~cm}^{-1} /$ atom $\mathrm{cm}^{-3}$ for $\mathrm{Cd}-\mathrm{He}$ determined by means of classical spectroscopy methods using a Fabry-Perot interferometer [37].

These and other tests performed during the course of this study have led us to the conclusion that the only cause of the decrease in the Lorentzian width $\gamma_{\mathrm{L}}$ with time is the decrease in the helium density number $N$ in the fluorescence cell due to the helium diffusion through the walls of quartz cell. The phenomenon of diffusion also known as permeation of helium through various glasses was discovered long time ago and corresponding permeation rates have been determined through different types of glasses, including quartz [38, 26]. It was established that these rates strongly depend on temperature. Using the temperature dependences described by Altemose [38] we can estimate that reduction of the quartz cell temperature from $724 \mathrm{~K}$ to $450 \mathrm{~K}$ decreases the helium permeation rate through the quartz walls by a factor of ten.

It is thus evident that in order to obtain reliable line shape parameters for $\mathrm{Cd}-\mathrm{He}$ the helium permeation should be minimized and this can be achieved if the cell temperature is sufficiently low. The choice of cell temperature becomes then a result of compromise between the intensity of the signal and the helium 
permeation through quartz walls. We found that the temperature $439 \mathrm{~K}$ fulfils the conditions of such a compromise and Fig. 7 shows the values of the Lorentzian width $\gamma_{\mathrm{L}}$ determined for this temperature from subsequent measurements of the shape of the $326.1 \mathrm{~nm} \mathrm{Cd}$ line perturbed by 230 Torr of helium. Like in Fig. 5 here the point zero in the timescale (in minutes) denotes the beginning of the first measurement. As can be seen, there is no systematic decrease in $\gamma_{\mathrm{L}}$ with time. We can thus conclude that for the cell temperature $439 \mathrm{~K}$ the errors in determination the line shape parameters due to the helium permeation through the quartz walls of cell are smaller than the statistical scatter of experimental data. All the results described below were obtained for the cell temperature $439 \mathrm{~K}$.

\subsection{Line shape parameters for $\mathrm{Cd}-\mathrm{He}$}

Figure 8a shows an example of an experimental shape of the $326.1 \mathrm{~nm} \mathrm{Cd}$ line perturbed by helium (at pressure 132 Torr at room temperature). The points represent the values of intensity measured at the cell temperature $439 \mathrm{~K}$. The full line in Fig. 8a is the best fit of the data to the AVP. We have also fitted our data to the ordinary VP, but this fit is not shown in Fig. 8a. In Fig. $8 \mathrm{~b}$ and $c$ we have

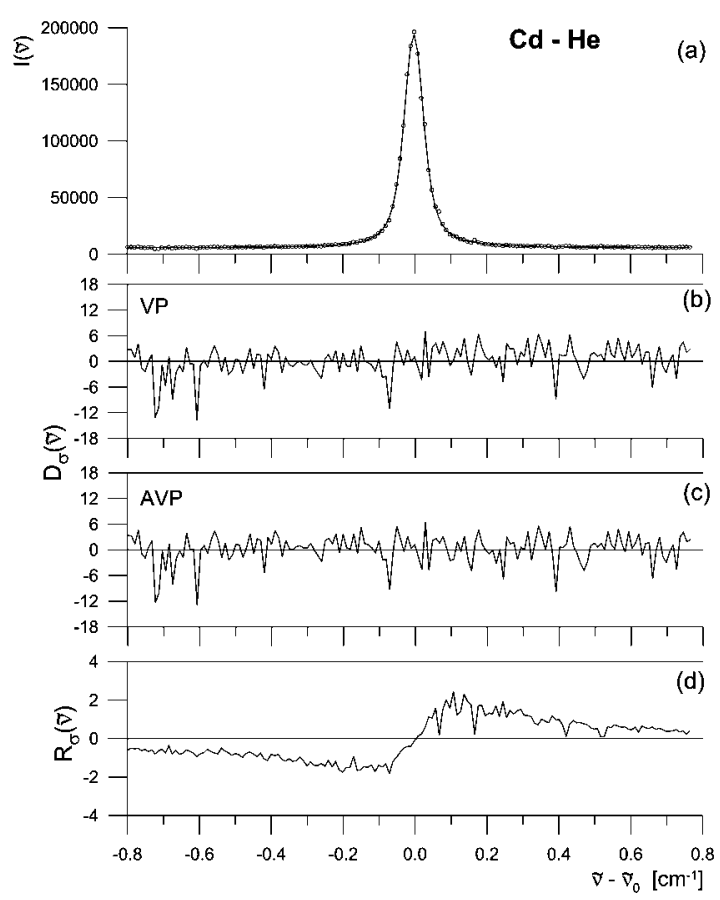

Fig. 8. The shape of the Cd $326.1 \mathrm{~nm}$ line perturbed by helium at pressure 132 Torr: (a) experimental points together with the best-fit AVP (full curve), (b) and (c) weighted residuals $D_{\sigma}(\widetilde{\nu})$ between experimental and fitted VP and AVP profiles, respectively, (d) differences $R(\widetilde{\nu})$. 
plotted the weighted residuals $D_{\sigma}^{\mathrm{VP}}(\widetilde{\nu})$ and $D_{\sigma}^{\mathrm{AVP}}(\widetilde{\nu})$, for the VP and AVP profiles, respectively, determined from Eq. (5).

As both residuals look very similar, in Fig. 8d we plotted the difference $R_{\sigma}(\widetilde{\nu})=D_{\sigma}^{\mathrm{AVP}}(\widetilde{\nu})-D_{\sigma}^{\mathrm{VP}}(\widetilde{\nu})$. We can see systematic departures from zero in the line core as well as on line wings which can be regarded as a manifestation of the line asymmetry. We can thus conclude that in the case of the $\mathrm{Cd}-\mathrm{He}$ system the collision-time asymmetry has a noticeable influence on the profile of the $326.1 \mathrm{~nm}$ line. Like for $\mathrm{Cd}-\mathrm{Ne}$ also for $\mathrm{Cd}-\mathrm{He}$ we found the higher intensity in blue wing of this line, in contrast to the heavy perturber case $[15-17]$ where the higher intensity in red wing was observed.

Figure 9 shows the values of the Doppler width $\gamma_{\mathrm{D}}$ of the $326.1 \mathrm{~nm} \mathrm{Cd}$ line perturbed by helium at $439 \mathrm{~K}$ determined from the best fit of the AVP to the experimental data, plotted against the pressure of He. As can be seen, there is practically no dependence of the Doppler width $\gamma_{\mathrm{D}}$ on the helium pressure. It confirms the statistical independence between the pressure and Doppler broadening for the $\mathrm{Cd}-\mathrm{He}$ system. The average Doppler width is found to be $43.5(2.8) \times 10^{-3} \mathrm{~cm}^{-1}$. This value agrees very well with theoretical Doppler width $43.1 \times 10^{-3} \mathrm{~cm}^{-1} \mathrm{cor}-$ responding to the cell temperature $T=439 \mathrm{~K}$.

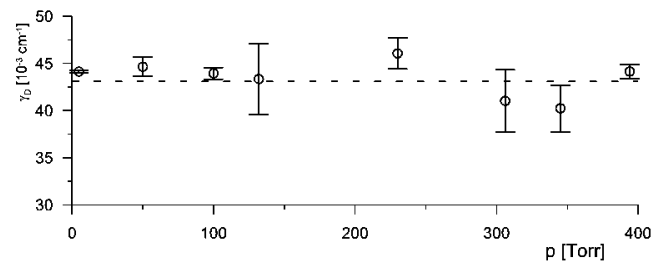

Fig. 9. Plot of the Doppler width $\gamma_{\mathrm{D}}$ of the $326.1 \mathrm{~nm} \mathrm{Cd}$ line perturbed by helium against the pressure of He. The widths $\gamma_{\mathrm{D}}$ are determined from the best fit of the AVP to the experimental data. Dashed line - Doppler width calculated from the cell temperature $(439 \mathrm{~K})$. Error bars indicate the value of the standard uncertainty.

Figure 10 shows the plots of the Lorentzian width $\gamma_{\mathrm{L}}$ determined from the best fit of $I_{\mathrm{AVP}}(\widetilde{\nu})$ to our experimental profiles against the helium density number $N$. It should be noted that each experimental point represents the mean value of a few measurements performed for a given pressure. The solid straight line represents the best fit of linear relation between $\gamma_{\mathrm{L}}$ and $N$. As can be seen, the experimental points were measured with high precision (small values of standard uncertainties) but show systematic departures from fitted straight line relation. From the slope of this linear relation the pressure broadening $\beta=\gamma_{\mathrm{L}} / N$ coefficient was determined and listed in Table I. The value of the pressure broadening coefficient obtained from the best fit procedure $\beta=(1.083 \pm 0.016) \times 10^{-20} \mathrm{~cm}^{-1} / \mathrm{atom} \mathrm{cm}^{-3}$ is lower than the value $\beta_{\mathrm{IFP}}=(1.22 \pm 0.03) \times 10^{-20} \mathrm{~cm}^{-1} /$ atom $\mathrm{cm}^{-3}$ resulting from interferometric measurements performed in similar temperature $(T=468 \mathrm{~K})$ [37]. 


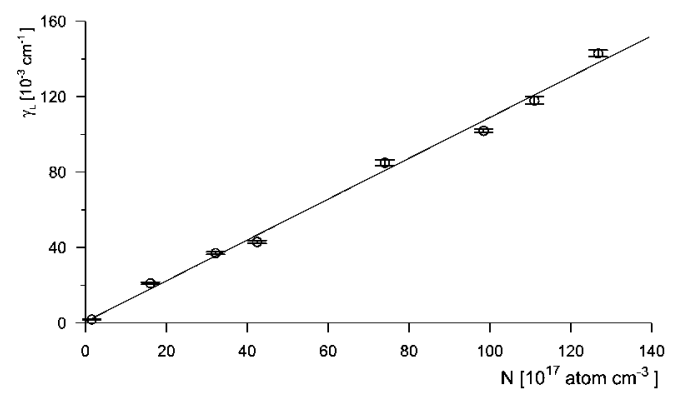

Fig. 10. Plot of the Lorentzian width $\gamma_{\mathrm{L}}$ of the $326.1 \mathrm{~nm} \mathrm{Cd}$ line determined from the best fit of the AVP to the experimental data against the helium density $N$. Error bars indicate the value of the standard uncertainty.

In order to explain this discrepancy we have determined the value of pressure broadening coefficient once again taking into account only the experimental points corresponding to perturber pressures $p<100$ Torr because this pressure range was the subject of FPI measurements [37]. The value of pressure broadening coefficient obtained in this way $\beta^{*}=(1.155 \pm 0.034) \times 10^{-20} \mathrm{~cm}^{-1} /$ atom $\mathrm{cm}^{-3}$ is in good agreement with $\beta_{\text {IFP }}$ value. A detailed analysis of $\gamma_{\mathrm{L}}$ values presented in Fig. 10 shows that for some helium pressures we obtained diminished values of Lorentzian width. As was shown above for the cell temperature $439 \mathrm{~K}$, we can neglect the diffusion of helium during the measurements, so the diminished $\gamma_{L}$ values may be due to helium leakage during the cut-off of the cell from the vacuum stand taking place through the very hot connection point.

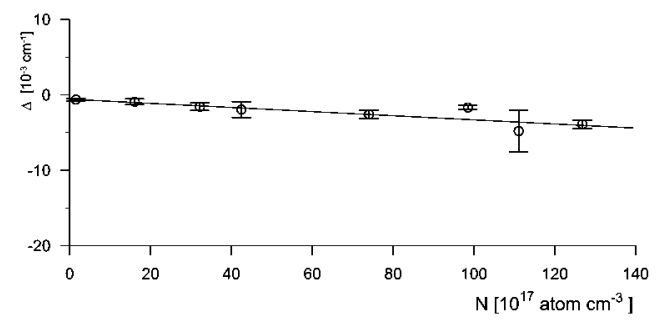

Fig. 11. Plot of the shift $\Delta$ of the $326.1 \mathrm{~nm}$ Cd line determined from the best fit of the AVP to the experimental data against the helium density $N$. Error bars indicate the value of the standard uncertainty.

Figure 11 shows the plots of the line shift $\Delta$ determined from the best fit of $I_{\mathrm{AVP}}(\widetilde{\nu})$ to our experimental profiles against the density number $N$ of helium. As it is seen, the line shift is towards the red and is linearly dependent on the helium density. From the slope of this linear relation the pressure shift $\delta=\Delta / N$ coefficient was determined and listed in Table I. Similarly as for the line width we determined the $\delta$ coefficient twice. First, using all experimental points, and second, taking into account only the experimental points corresponding to helium 
pressures $p<100$ Torr. As can be seen from Table I, these two values are very close.

In Fig. 12 we plotted the values of asymmetry parameters $\chi$ determined from the best fit of $I_{\mathrm{AVP}}(\widetilde{\nu})$ to our experimental profiles as the function of density number $N$ of helium. From the slopes of the linear fit including, first, all and then only $p<100$ Torr experimental points we determined two values of asymmetry coefficients $\kappa=\chi / N$ and listed them in Table II. As it is seen, these two $\kappa$ values differ by a factor about 3 . It should be noted that for $\mathrm{Cd}-\mathrm{He}$ system, like for $\mathrm{Cd}-\mathrm{Ne}$ we observe the positive sign of asymmetry coefficient $(\kappa>0)$ while the line shift is towards the red $(\delta<0)$. The analogous case was observed by the Lewis group for $422.7 \mathrm{~nm} \mathrm{Ca}$ line perturbed by $\mathrm{He}[3-5]$. The positive $\kappa$ values were also found by Romalis et al. [6] for the rubidium $D_{1}$ and $D_{2}$ lines perturbed by ${ }^{3} \mathrm{He}$ and ${ }^{4} \mathrm{He}$, but in their case the line shift was towards the blue $(\delta>0)$. Keeping in mind the possible errors of perturber concentration, especially for higher helium pressures we can thus conclude that more reliable are the values of pressure coefficients corresponding to $p<100$ Torr pressure range, listed in second row of Table II.

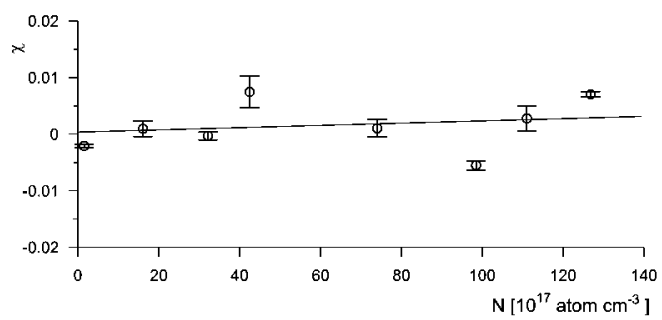

Fig. 12. Plot of the asymmetry parameter $\chi$ determined from the best fit of AVP to the experimental data against the helium density $N$. Error bars indicate the value of the standard uncertainty.

TABLE II

Experimental values of the $\beta, \delta$ (in units $10^{-20} \mathrm{~cm}^{-1} /$ at. $\mathrm{cm}^{-3}$ ) and $\kappa$ (in units $10^{-21} /$ at. $\mathrm{cm}^{-3}$ ) for Cd-He system. Numbers in parentheses are the values of standard uncertainty. Details in the text.

\begin{tabular}{l|c|c|c}
\hline \hline Fitted profile & $\beta$ & $\delta$ & $\kappa$ \\
\hline AVP & $1.083(16)$ & $-0.027(5)$ & $0.20(18)$ \\
\hline AVP $(p<100$ Torr $)$ & $1.155(34)$ & $-0.031(9)$ & $0.62(34)$
\end{tabular}

The experimental values of pressure broadening $\beta$, shift $\delta$ and asymmetry $\kappa$ coefficients (corresponding to $p<100$ Torr pressure range) determined for $\mathrm{Cd}-\mathrm{He}$ are listed in Table III together with $\beta$ and $\delta$ coefficients determined from our 
TABLE III

Comparison of experimental values of the $\beta, \delta$ (in units $10^{-20} \mathrm{~cm}^{-1} /$ at. $\mathrm{cm}^{-3}$ ) and $\kappa$ (in units $10^{-21} /$ at. $\mathrm{cm}^{-3}$ ) coefficients for $\mathrm{Cd}-\mathrm{He}$ system (determined for helium pressure $p<100$ Torr) with those calculated for different interatomic potentials. For experimental data the values of standard uncertainty are given.

\begin{tabular}{l|c|c|c}
\hline \hline Experimental values & $\beta_{\text {exp }}$ & $\delta_{\text {exp }}$ & $\kappa_{\exp }$ \\
\hline This work & $1.155(34)$ & $-0.031(9)$ & $0.62(34)$ \\
Bielski et al. [22] & $1.22(3)$ & $0.02(2)$ & - \\
Dietz et al. [35] & $1.16(8)$ & $-0.051(23)$ & - \\
Evdokimov et al. [36] & $3.10(28)$ & $0.20(2)$ & - \\
\hline Calculated values & $\beta_{\text {calc }}$ & $\delta_{\text {calc }}$ & $\kappa_{\text {calc }}$ \\
\hline Czuchaj-Stoll [19] & 1.094 & 0.059 & 0.083 \\
Morse & 0.726 & -0.286 & -0.07 \\
van der Waals (H-F) & 0.853 & -0.310 & -0.14 \\
van der Waals (CA) & 0.664 & -0.241 & -0.01
\end{tabular}

earlier [37] interferometric measurements as well as the with the values obtained by Dietz et al. [30] and Evdokimov et al. [39].

As it can be seen from Table III, the values of pressure broadening $\beta$ and shift $\delta$ coefficients obtained in the course of the present work are in good agreement with the values obtained from both the interferometric [37] and Dietz et al. [36] measurements. The negative value of pressure shift coefficient obtained in the present work agrees with the value measured by Dietz et al. [36]. Our earlier interferometric measurements showed practically no line shift for Cd-He system. However, within the standard uncertainty all the above $\delta$ values agree with each other. Our experimental data also confirm the observation made by Dietz that for $\mathrm{Cd}-\mathrm{He}$ system the line shape parameters are independent of temperature. On the other hand, our experimental values for $\beta$ and $\delta$ coefficients disagree with the values given by Evdokimov et al. [39].

\section{Conclusion}

In the present work we have shown that the asymmetric Voigt profile gives a correct description of our experimental profiles of the ${ }^{114} \mathrm{Cd} 326.1 \mathrm{~nm}$ line perturbed by neon and by helium.

In order to interpret our experimental data we calculated the theoretical values of pressure broadening $\beta$ and shift $\delta$ coefficients on the basis of the adiabatic phase-shift theory with straight-line trajectories using Eq. (3) from Ref. [15]. Our calculations were performed for the Czuchaj and Stoll [19] numerical potential as well as for an empirical potential derived from experimental data in the form of 
the Morse potential with the spectroscopic constants determined recently by Koperski and Czajkowski [20]. We also calculated the values of $\beta$ and $\delta$ assuming the interaction potential in the van der Waals form, using the values of $C_{6}$ force constants calculated in the Coulomb approximation (CA) and using Hartree-Fock $(\mathrm{H}-\mathrm{F})$ wave functions. The values of $\beta$ and $\delta$ coefficients evaluated for these potentials are listed in Table $\mathbf{I}$ for $\mathrm{Cd}-\mathrm{Ne}$ and Table III for $\mathrm{Cd}-\mathrm{He}$ and marked as "Czuchaj-Stoll [19]", "Morse", "van der Waals (CA)" and "van der Waals (H-F)", respectively.

As can be seen from Tables I and III, there is generally poor agreement between the experimental and theoretical values both for $\beta$ and $\delta$ coefficients. For $\mathrm{Cd}-\mathrm{He}$ our experimental value of pressure broadening $\beta$ coefficient is in quantitative agreement with theoretical value calculated for Czuchaj-Stoll [19] potential, but for the line shift this potential gives rise to opposite sign. For $\mathrm{Cd}-\mathrm{Ne}$ experimental values of $\beta$ and $\delta$ are close to theoretical values obtained for the Morse potential.

In Tables I and III we also listed the value of asymmetry coefficient $\kappa$ calculated for all of the above potentials using Eq. (10) from Ref. [15]. For $\mathrm{Cd}-\mathrm{He}$ the only qualitative agreement between experimental and calculated values of $\kappa$ coefficients is for the Czuchaj-Stoll [19] potential, while the other potentials give rise to opposite (negative) value of $\kappa$ coefficient.

The comparison of pressure broadening, shift and asymmetry coefficients determined in this experiment with coefficients calculated on the basis of the adiabatic semiclassical approach shows poor agreement between experimental and calculated values obtained for numerical potentials calculated by Czuchaj and Stoll [19] as well as for empirical potentials of the Morse and Lennard-Jones form derived from experimental data.

The opposite signs of pressure shift and asymmetry coefficients indicate that the interaction potential cannot be assumed to have the van der Waals form since in this case negative sign is predicted by the theory both for the shift and asymmetry.

We can thus conclude that new calculations of the interatomic potentials for $\mathrm{Cd}-\mathrm{Ne}$ and $\mathrm{Cd}-\mathrm{He}$ system are necessary to explain the experimental data on the broadening, shift and asymmetry of the Cd intercombinational line shape.

\section{Acknowledgment}

This work was supported by a grant No. 5 PO3B 06620 (354/PO3/2001/20) from the State Committee for Scientific Research. 


\section{References}

[1] S.Y. Chen, M. Takeo, Rev. Mod. Phys. 29, 20 (1957).

[2] N. Allard, J. Kielkopf, Rev. Mod. Phys. 54, 1103 (1982).

[3] M. Harris, E.L. Lewis, D. McHugh, I. Shannon, J. Phys. B 17, L661 (1984); 19, 3207 (1986).

[4] I. Shannon, M. Harris, P.R. McHugh, E.L. Lewis, J. Phys. B 19, 1409 (1986).

[5] E.L. Lewis, Spectral Line Shapes, Vol. 5, Ed. J. Szudy, Ossolineum Publ., Wrocław 1989 , p. 485.

[6] M.V. Romalis, E. Miron, G.D. Cates, Phys. Rev. A 56, 4569 (1997).

[7] T.W. Mossberg, R. Kachru, K.P. Leung, E. Whittaker, S.R. Hartmall, Spectral Line Shapes, Eds. B. Wende, W. de Gruyter, Berlin 1981, p. 1093.

[8] E. Fermi, Nuovo Cimento 11, 157 (1934).

[9] A. Omont, J. Phys. (Paris) 38, 1343 (1977).

[10] R.S. Trawiński, A. Bielski, J. Szudy, J. Wolnikowski, Acta Phys. Pol. A 86, 333 (1994).

[11] R.S. Dygdała, W. Lisicki, J. Szudy, J. Phys. B 20, 3001 (1981).

[12] E. Lisicki, A. Bielski, J. Szudy, Z. Naturforsch. A 36, 807 (1981).

[13] B. Cheron, R. Sheps, A. Gallagher, Phys. Rev. A 15, 651 (1977).

[14] R.S. Dygdała, R. Bobkowski, E. Lisicki, J. Phys. B 22, 1563 (1989).

[15] A. Bielski, R. Ciuryło, J. Domysławska, D. Lisak, R.S. Trawiński, J. Szudy, Phys. Rev. A 62, 032511 (2000).

[16] R.S. Trawiński, A. Bielski, D. Lisak, Acta Phys. Pol. A 99, 243 (2001).

[17] A. Bielski, D. Lisak, R.S. Trawiński, Eur. Phys. J. D 14, 27 (2001).

[18] A. Bielski, R. Ciuryło, J. Domysławska, D. Lisak, R.S. Trawiński, J. Szudy, Acta Phys. Pol. B 33, 2267 (2002).

[19] E. Czuchaj, H. Stoll, Chem. Phys. 248, 1 (1999).

[20] J. Koperski, M. Czajkowski, J. Chem. Phys. 109, 459 (1998); Eur. Phys. J. D 10, $363(2000)$.

[21] A. Bielski, R. Ciuryło, J. Domysławska, D. Lisak, R.S. Trawiński, J. Wolnikowski, Acta Phys. Pol. A 97, 1003 (2000).

[22] A. Bielski, S. Brym, R. Ciuryło, J. Domysławska, E. Lisicki, R.S. Trawiński, J. Phys. B 27, 5863 (1994).

[23] R.E. Walkup, A. Spielfiedel, D. Ely, W.D. Phillips, D.E. Pritchard, J. Phys. B 14, 1953 (1981).

[24] J. Ward, J. Cooper, E.W. Smith, J. Quant. Spectrosc. Radiat. Transfer 14, 555 (1974) 
[25] R. Ciuryło, Phys. Rev. 58, 1029 (1998).

[26] A. Roth, Vacuum Technology, North-Holland Publ., Amsterdam 1976.

[27] P.W. Anderson, J.D. Talman, Bell Teleph. Syst. Tech. Publ. No. 3117, University of Pittsburg, USA 1955.

[28] J. Szudy, W.E. Baylis, J. Quant. Spectrosc. Radiat. Transfer 15, 641 (1975); 17, 681 (1977).

[29] G. Peach, J. Phys. B 17, 2599 (1984); B.N.I. Al-Saqabi, G. Peach, J. Phys. B 20, 1175 (1987).

[30] R. Ciuryło, J. Szudy, R.S. Trawiński, J. Quant. Spectrosc. Radiat. Transfer 57, 551 (1997).

[31] D.W. Marquardt, J. Soc. Industr. Appl. Math. 11, 431 (1963).

[32] P.R. Berman, J. Quant. Spectrosc. Radiat. Transfer 12, 1331 (1972).

[33] S. Brym, R. Ciuryło, R. S. Trawiński, A. Bielski, Phys. Rev. A 56, 4501 (1997).

[34] S. Brym, J. Domysławska, Phys. Scr. 52, 511 (1995).

[35] S. Brym, R. Ciuryło, E. Lisicki, R.S. Trawiński, Phys. Scr. 56, 541 (1996).

[36] K.J. Dietz, P. Dabkiewicz, H.J. Kluge, T. Kühl, H.A. Schuessler, J. Phys. B 13, 2749 (1980).

[37] A. Bielski, S. Brym, R. Ciuryło, J. Domysławska, E. Lisicki, R.S. Trawiński, J. Phys. B 27, 5863 (1994).

[38] V.O. Altemose, J. Appl. Phys. 32, 1309 (1961).

[39] Y.U. Evdokimov, N.I. Kaliteyevskii, M.P. Chaika, Opt. Spektrosk. 27, 187 (1969). 